\title{
Exhaustive vacation queue with dependent arrival and service processes *
}

\author{
Gábor Horváth ${ }^{1,2}$, Zsolt Saffer ${ }^{3}$, and Miklós Telek ${ }^{1,2}$ \\ ${ }^{1}$ Budapest University of Technology and Economics, Hungary \\ ${ }^{2}$ MTA-BME Information systems research group, Hungary \\ ${ }^{3}$ No affiliation currently \\ \{hgabor,saffer,telek\}@webspn.hit.bme.hu
}

\begin{abstract}
This paper presents a more general class of MAP/MAP/1 exhaustive vacation queue, in which the Markov modulated arrival and service processes are dependent. This model class requires the evaluation of the busy period of quasi birth death process with arbitrary initial level, which is a new analysis element.

The model is analyzed by applying matrix analytic methods for the underlying quasi birth death process. The main result of the paper is the probability-generating function of the number of jobs in the system. Finally, a numerical example provides an insight into the behavior of the model.
\end{abstract}

Keywords: vacation queue, MAP, dependent arrival and service process, QBD, matrix analytic methods, stationary analysis.

\section{Introduction}

The importance of vacation queues comes for their diverse application fields: modeling various computer systems, telecommunication protocols, manufacturing, logistics, etc. For details on analysis works on vacation models and their generalizations the reader is referred to the recent surveys [5] and [10].

Due to the versatility of the Markovian Arrival Process (MAP) [7], vacation queues with MAP input and general service times have also been investigated in several past papers $[4,8,9]$. Only a few discrete-time models have been investigated, in which both the arrival and the service processes are Markovian. $\mathrm{MAP} / \mathrm{PH} / 1$ vacation models have been analyzed by A.-S. Alpha in $[1,2]$ and by C. Goswami and N. Selvaraju in [3].

In this paper we consider a more general class of exhaustive vacation queues with dependent Markov modulated arrival and service processes. This model class requires the introduction of a new analysis element, the evaluation of the busy period of quasi birth death (QBD) processes with arbitrary initial level. We provide the expression of the probability-generating function (PGF) of the

\footnotetext{
^ The authors thank the support of the OTKA K101150 project.
} 
number of jobs in the system. In the last part of the paper we provide a numerical example and investigate the effects of different vacation distributions on the mean number of jobs.

\section{Model description}

We consider the dependent MAP/MAP/1 exhaustive vacation queue. The model falls in the class of single server FCFS queue with multiple vacations and exhaustive discipline [10]. According to the rule of exhaustive service discipline the server serves the jobs in the queue until it gets idle, then the server leaves for vacation for an independent and identically distributed random amount of time. If the queue is idle at the end of the vacation the server leaves for a new vacation, otherwise it starts serving the jobs in the queue. The random vacation time, its probability density function (pdf) and its Laplace transform (LT) are denoted by $\tilde{\sigma}, \sigma(t)$ and $\sigma^{*}(s)=E\left(e^{-s \tilde{\sigma}}\right)$, respectively.

The arrivals and services are characterized by seven matrices: $\mathbf{L}_{\mathbf{v}}, \mathbf{F}_{\mathbf{v}}, \mathbf{B}_{\mathbf{s}}, \mathbf{L}_{\mathbf{s}}, \mathbf{F}_{\mathbf{s}}, \boldsymbol{\Pi}_{\mathbf{v s}}$ and $\boldsymbol{\Pi}_{\mathbf{s v}}$.

- During the vacations the arrivals are given by a MAP, where the entries of $\mathbf{L}_{\mathbf{v}}$ are the rates of transitions without a job arrival, and the entries of $\mathbf{F}_{\mathbf{v}}$ are the rates of transitions that are accompanied by a job arrival. Matrix $\mathbf{L}_{\mathbf{v}}+\mathbf{F}_{\mathbf{v}}$ is therefore the generator of the continuous time Markov chain (CTMC) with $N_{v}$ states which modulates the arrivals during vacation.

- When the server serves the jobs, the queue behaves as a quasi birth-death (QBD) [6] process, there the matrices $\mathbf{B}_{\mathbf{s}}, \mathbf{L}_{\mathbf{s}}$ and $\mathbf{F}_{\mathbf{s}}$ contains the transition rates associated with a service completion, without service completion and job arrival, with a job arrival, respectively. In this case the generator of the modulating CTMC is $\mathbf{B}_{\mathbf{s}}+\mathbf{L}_{\mathbf{s}}+\mathbf{F}_{\mathbf{s}}$, and it has $N_{s}$ states.

- The transition between the vacation and service periods is given by $N_{v} \times$ $N_{s}$ stochastic matrix $\boldsymbol{\Pi}_{\mathbf{v s}}$, whose entries are the probabilities of the state transitions occurring at the end of the vacation period. The $N_{s} \times N_{v}$ matrix $\boldsymbol{\Pi}_{\mathbf{s v}}$ has a similar role, holding the probabilities of phase transitions when the service period ends and a vacation starts.

This is a general model which covers a number of special cases, e.g., the $\mathrm{MAP} / \mathrm{PH} / 1$ vacation queue and the $\mathrm{MAP} / \mathrm{MAP} / 1$ vacation queue.

The stability of the model is determined by the stationary drift of the QBD during service [6]. Hence the necessary and sufficient condition of the stability of this vacation model is

$$
\alpha_{s} \mathbf{F}_{\mathbf{s}} \mathbb{1}-\alpha_{s} \mathbf{B}_{\mathbf{s}} \mathbb{1}<0
$$

where $\alpha_{s}$ is the solution of the linear system $\alpha_{s}\left(\mathbf{B}_{\mathbf{s}}+\mathbf{L}_{\mathbf{s}}+\mathbf{F}_{\mathbf{s}}\right)=\mathbf{0}, \alpha_{s} \mathbb{1}=1$, and $\mathbb{1}$ denotes the column vector of ones. 


\section{The number of jobs in the system}

To characterize the number of jobs in the system, let us introduce the two dimensional process $\mathcal{X}(t)=\{\mathcal{N}(t), \mathcal{J}(t), t \geq 0\}$, where $\mathcal{N}(t)$ denotes the number of jobs (also referred to as levels) and $\mathcal{J}(t)$ denotes the state of the modulating CTMC (also referred to as phase) at time $t$. For the analysis of $\mathcal{X}(t)$ the evolution of the queue is divided to cycles, as shown in Figure 1. Each cycle starts with a vacation period, which is followed by a service period, and the cycle ends when the last job leaves the system. Note that a cycle can also be degenerate: if no jobs arrive during the vacation period, there is no service period (see cycle $i-1$ in Figure 1).

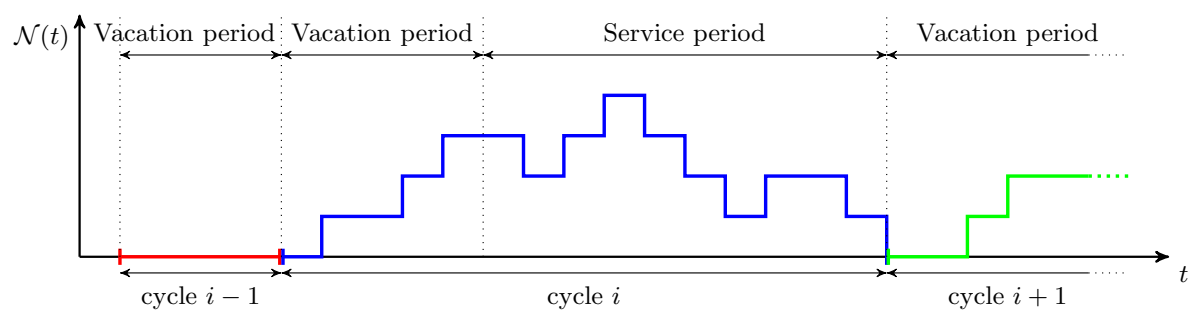

Fig. 1. Cycles in the evolution of the queue

The stationary probability that there are $\ell(\ell \geq 1)$ jobs in the system is proportional to $M_{\ell}$, the mean time spent at level $\ell$ in a stationary cycle.

$$
M_{\ell}=\underbrace{\int_{u=0}^{\infty} \sigma(u) \int_{t=0}^{u} \beta \mathbf{P}_{\ell}^{(\mathbf{v})}(t) \mathbb{1} d t d u}_{M_{\ell}^{(v)}}+\underbrace{\int_{u=0}^{\infty} \sigma(u) \sum_{m=1}^{\infty} \beta \mathbf{P}_{m}^{(\mathbf{v})}(u) \mathbf{\Pi}_{\mathbf{v s}} \mathbf{H}_{m, \ell} \mathbb{1} d u}_{M_{\ell}^{(s)}},
$$

where row vector $\beta$ of size $N_{v}$ is the stationary phase distribution at the beginning of the vacation period, matrix $\mathbf{P}_{\ell}^{(\mathbf{v})}(t)$ characterizes the number of arrivals up to time $t$ during the vacation period, defined as

$$
\left[\mathbf{P}_{\ell}^{(\mathbf{v})}(t)\right]_{i, j}=P(\mathcal{N}(t)=\ell, \mathcal{J}(t)=j, \tilde{\sigma}>t \mid \mathcal{N}(0)=0, \mathcal{J}(0)=i),
$$

and $\left[\mathbf{H}_{m, \ell}\right]_{i, j}$ is the mean times spent in level $\ell$ and phase $j$ in the service period starting from level $m$ and phase $i$. The first and second term of (2), M(v) and $\mathrm{M}(\mathrm{s})$, correspond to the vacation and service period, respectively. Closed form formulas are provided for both in the next subsections. From $M_{\ell}$ the stationary distribution of $\mathcal{N}(t)$ is obtained by normalization, $q_{\ell}=\lim _{t \rightarrow \infty} P(\mathcal{N}(t)=\ell)=$ $M_{\ell} / \sum_{k} M_{k}$.

The evolution of the number of jobs during the vacation period The evolution of the number of jobs during the vacation period resembles to the 
counting process of a MAP given by matrices $\mathbf{L}_{\mathbf{v}}, \mathbf{F}_{\mathbf{v}}$. Thus, for matrices $\mathbf{P}_{\ell}^{(\mathbf{v})}(t)$ we have

$$
\begin{aligned}
\frac{d}{d t} \mathbf{P}_{\ell}^{(\mathbf{v})}(t) & =\mathbf{P}_{\ell-1}^{(\mathbf{v})}(t) \mathbf{F}_{\mathbf{v}}+\mathbf{P}_{\ell}^{(\mathbf{v})}(t) \mathbf{L}_{\mathbf{v}}, \text { for } \ell>0, \\
\frac{d}{d t} \mathbf{P}_{0}^{(\mathbf{v})}(t) & =\mathbf{P}_{0}^{(\mathbf{v})}(t) \mathbf{L}_{\mathbf{v}}
\end{aligned}
$$

with initial condition $\mathbf{P}_{\ell}^{(\mathbf{v})}(0)=\delta_{0, \ell} \mathbf{I}$, where $\delta$ denotes the Kronecker delta (that is $\delta_{i i}=1, \delta_{i j}=0$ for $i \neq j$ ). Similar to [6, Sec. 3], multiplying the $\ell$ th equation by $z^{\ell}$, summing up and solving the differential equation gives the generating function

$$
\mathbf{P}^{(\mathbf{v}) *}(z, t)=\sum_{\ell=0}^{\infty} z^{\ell} \mathbf{P}_{\ell}^{(\mathbf{v})}(t)=e^{\left(\mathbf{L}_{\mathbf{v}}+z \mathbf{F}_{\mathbf{v}}\right) t}
$$

The mean time spent in different levels during the service period As a new contributions of the paper we derive matrix $\mathbf{H}_{m, \ell}$, which is the mean time spent in various phases of level $\ell$ starting from level $m$ in a QBD characterized by matrices $\mathbf{B}_{\mathbf{s}}, \mathbf{L}_{\mathbf{s}}$ and $\mathbf{F}_{\mathbf{s}}$. It is known that the mean time spent at different phases of level $\ell$ starting from level 0 before returning to level 0 is given by $\mathbf{R}^{\ell}$ $[6]$.

But, in our vacation queue the starting level after a vacation is not 0 , but the number of arrivals during the vacation, which is denoted by $m$. According to our best knowledge, this measure has not been investigated yet.

For $m>0$, we define matrix $\mathbf{P}_{m, \ell}^{(\mathbf{s})}$ corresponding to the service period as

$$
\left[\mathbf{P}_{m, \ell}^{(\mathbf{s})}(t)\right]_{i, j}=P(\Theta>u+t, \mathcal{N}(u+t)=\ell, \mathcal{J}(u+t)=j \mid \mathcal{N}(u)=m, \mathcal{J}(u)=i, \tilde{\sigma}=u)
$$

where $u$ marks the beginning and $\Theta$ marks the end of the service period, thus $\Theta=\min \{t: \mathcal{N}(u+t)=0\}$. For $\ell>1$ matrix $\mathbf{P}_{m, \ell}^{(\mathbf{s})}(t)$ satisfies

$$
\frac{d}{d t} \mathbf{P}_{m, \ell}^{(\mathbf{s})}(t)=\mathbf{P}_{m, \ell-1}^{(\mathbf{s})}(t) \mathbf{F}_{\mathbf{s}}+\mathbf{P}_{m, \ell}^{(\mathbf{s})}(t) \mathbf{L}_{\mathbf{s}}+\mathbf{P}_{m, \ell+1}^{(\mathbf{s})}(t) \mathbf{B}_{\mathbf{s}}
$$

and for $\ell=1$ we have

$$
\frac{d}{d t} \mathbf{P}_{m, 1}^{(\mathbf{s})}(t)=\mathbf{P}_{m, 1}^{(\mathbf{s})}(t) \mathbf{L}_{\mathbf{s}}+\mathbf{P}_{m, 2}^{(\mathbf{s})}(t) \mathbf{B}_{\mathbf{s}}
$$

with initial values $\mathbf{P}_{m, \ell}^{(\mathbf{s})}(0)=\delta_{m, \ell} \mathbf{I}$. We are interested in the mean time spent in different states during the busy period, that is $\mathbf{H}_{m, \ell}=\int_{t=0}^{\infty} \mathbf{P}_{m, \ell}^{(\mathbf{s})}(t) d t$. Integrating the differential equation (7) and (8) from $t=0$ to $\infty$ we get

$$
\begin{aligned}
& \mathbf{P}_{m, \ell}^{(\mathbf{s})}(\infty)-\mathbf{P}_{m, \ell}^{(\mathbf{s})}(0)=\mathbf{H}_{m, \ell-1} \mathbf{F}_{\mathbf{s}}+\mathbf{H}_{m, \ell} \mathbf{L}_{\mathbf{s}}+\mathbf{H}_{m, \ell+1} \mathbf{B}_{\mathbf{s}}, \quad \text { for } \ell>1 \\
& \mathbf{P}_{m, 1}^{(\mathbf{s})}(\infty)-\mathbf{P}_{m, 1}^{(\mathbf{s})}(0)=\mathbf{H}_{m, 1} \mathbf{L}_{\mathbf{s}}+\mathbf{H}_{m, 2} \mathbf{B}_{\mathbf{s}} .
\end{aligned}
$$


These two equations and the initial value $\mathbf{P}_{m, \ell}^{(\mathbf{s})}(0)$ lead to four different cases, in general: (1) when $\ell=1$, (2) when $1<\ell<m$, (3) when $1<\ell=m$, finally, (4) when $\ell>m$. The corresponding equations are

$$
\begin{aligned}
-\delta_{m, 1} \mathbf{I} & =\mathbf{H}_{m, 1} \mathbf{L}_{\mathbf{s}}+\mathbf{H}_{m, 2} \mathbf{B}_{\mathbf{s}}, & & \\
\mathbf{0} & =\mathbf{H}_{m, \ell-1} \mathbf{F}_{\mathbf{s}}+\mathbf{H}_{m, \ell} \mathbf{L}_{\mathbf{s}}+\mathbf{H}_{m, \ell+1} \mathbf{B}_{\mathbf{s}}, & & \text { for } 1<\ell<m, \\
-\mathbf{I} & =\mathbf{H}_{m, m-1} \mathbf{F}_{\mathbf{s}}+\mathbf{H}_{m, m} \mathbf{L}_{\mathbf{s}}+\mathbf{H}_{m, m+1} \mathbf{B}_{\mathbf{s}}, & & \text { for } m>1, \\
\mathbf{0} & =\mathbf{H}_{m, \ell-1} \mathbf{F}_{\mathbf{s}}+\mathbf{H}_{m, \ell} \mathbf{L}_{\mathbf{s}}+\mathbf{H}_{m, \ell+1} \mathbf{B}_{\mathbf{s}}, & & \text { for } \ell>m,
\end{aligned}
$$

where $\delta$ denotes the Kronecker delta again. The solution of (11)-(14) is given by a matrix-geometric combination

$$
\begin{array}{ll}
\mathbf{H}_{m, \ell}=\mathbf{\Phi} \mathbf{R}^{\ell-1}+\mathbf{\Psi} \mathbf{S}^{m-\ell}, & \text { for } 1 \leq \ell \leq m, \\
\mathbf{H}_{m, \ell}=\mathbf{H}_{m, m} \mathbf{R}^{\ell-m}, & \text { for } 1 \leq m<\ell,
\end{array}
$$

where matrices $\mathbf{R}$ and $\mathbf{S}$ are obtained such that the regular equations (12) and (14) are satisfied for any $\boldsymbol{\Psi}$ and $\boldsymbol{\Phi}$. R and $\mathbf{S}$ are the minimal non-negative solutions to the quadratic equations [6, Sec. 10]

$$
\mathbf{0}=\mathbf{F}_{\mathbf{s}}+\mathbf{R L}_{\mathbf{s}}+\mathbf{R}^{2} \mathbf{B}_{\mathbf{s}}, \quad \mathbf{0}=\mathbf{B}_{\mathbf{s}}+\mathbf{S L}_{\mathbf{s}}+\mathbf{S}^{2} \mathbf{F}_{\mathbf{s}}
$$

Matrices $\boldsymbol{\Psi}$ and $\boldsymbol{\Phi}$ are obtained from the solution of the irregular equations (11) and (13) as

$$
\begin{aligned}
\mathbf{0} & =\boldsymbol{\Phi}\left(\mathbf{L}_{\mathbf{s}}+\mathbf{R B}_{\mathbf{s}}\right)+\mathbf{\Psi}\left(\mathbf{S}^{m-1} \mathbf{L}_{\mathbf{s}}+\mathbf{S}^{m-2} \mathbf{B}_{\mathbf{s}}\right), \\
-\mathbf{I} & =\mathbf{\Phi} \mathbf{R}^{m-2} \underbrace{\left(\mathbf{F}_{\mathbf{s}}+\mathbf{R L}_{\mathbf{s}}+\mathbf{R}^{2} \mathbf{B}_{\mathbf{s}}\right)}_{\mathbf{0}}+\mathbf{\Psi}\left(\mathbf{S F}_{\mathbf{s}}+\mathbf{L}_{\mathbf{s}}+\mathbf{R B} \mathbf{B}_{\mathbf{s}}\right) .
\end{aligned}
$$

The solution of $\boldsymbol{\Psi}$ and $\boldsymbol{\Phi}$ are

$$
\begin{aligned}
\boldsymbol{\Psi} & =\left(-\mathbf{S F}_{\mathbf{s}}-\mathbf{L}_{\mathbf{s}}-\mathbf{R B}_{\mathbf{s}}\right)^{-1} \\
\boldsymbol{\Phi} & =\boldsymbol{\Psi}\left(\mathbf{S}^{m-1} \mathbf{L}_{\mathbf{s}}+\mathbf{S}^{m-2} \mathbf{B}_{\mathbf{s}}\right)\left(\mathbf{L}_{\mathbf{s}}+\mathbf{R} \mathbf{B}_{\mathbf{s}}\right)^{-1} \\
& =-\mathbf{\Psi} \mathbf{S}^{m} \mathbf{F}_{\mathbf{s}}\left(\mathbf{L}_{\mathbf{s}}+\mathbf{R} \mathbf{B}_{\mathbf{s}}\right)^{-1}=-\mathbf{\Psi} \mathbf{S}^{m} \mathbf{R}
\end{aligned}
$$

where we exploited various identities of the fundamental matrices of QBDs. Finally using the expressions of $\mathbf{H}_{m, \ell}$ from (16) and (15) as well as (20) and (21) we get

$$
\begin{array}{rlr}
\mathbf{H}_{m, \ell}=\underbrace{-\boldsymbol{\Psi} \mathbf{S}^{m} \mathbf{R}^{\ell}}_{\text {term } 1}+\underbrace{\mathbf{\Psi} \mathbf{R}^{\ell-m}}_{\text {term } 2}, & \text { for } 1 \leq m \leq \ell, \\
\mathbf{H}_{m, \ell}=\underbrace{-\mathbf{\Psi} \mathbf{S}^{m} \mathbf{R}^{\ell}}_{\text {term } 1}+\underbrace{\mathbf{\Psi} \mathbf{S}^{m-\ell}}_{\text {term } 3}, & \text { for } 1 \leq \ell<m .
\end{array}
$$


The mean time spent at each level in a stationary cycle By applying (6) in the first term of (2), its generating function, $M_{\ell}^{(v)}$, can be expressed as

$$
\begin{aligned}
M^{(v) *}(z) & =\sum_{\ell=0}^{\infty} z^{\ell} M_{\ell}^{(v)}=\beta \int_{u=0}^{\infty} \sigma(u) \int_{t=0}^{u} e^{\left(\mathbf{L}_{\mathbf{v}}+z \mathbf{F}_{\mathbf{v}}\right) t} \mathbb{1} d t d u \\
& =\beta \int_{u=0}^{\infty} \sigma(u)\left(\mathbf{I}-e^{\left(\mathbf{L}_{\mathbf{v}}+z \mathbf{F}_{\mathbf{v}}\right) u}\right)\left(-\mathbf{L}_{\mathbf{v}}-z \mathbf{F}_{\mathbf{v}}\right)^{-1} \mathbb{1} d u \\
& =\beta\left(\mathbf{I}-\sigma^{*}\left(\mathbf{L}_{\mathbf{v}}+z \mathbf{F}_{\mathbf{v}}\right)\right)\left(-\mathbf{L}_{\mathbf{v}}-z \mathbf{F}_{\mathbf{v}}\right)^{-1} \mathbb{1}
\end{aligned}
$$

where $\sigma^{*}(\mathbf{M})$ with square matrix $\mathbf{M}$ is defined by $\int_{u=0}^{\infty} \sigma(u) e^{\mathbf{M} u} d u$.

Lemma 1. For any row vector $x$ of size $N_{v}$, matrix $\mathbf{X}$ of size $N_{v} \times N_{s}$ and matrix $\mathbf{Y}$ of size $N_{s} \times N_{s}$, if the infinite sum exists we have

$$
\sum_{m=0}^{\infty} x \mathbf{P}_{m}^{(\mathbf{v})}(t) \mathbf{X} \mathbf{Y}^{m}=v e c^{T}\left\langle\mathbf{X}^{T}\right\rangle e^{\left(\mathbf{L}_{\mathbf{v}}{ }^{T} \otimes \mathbf{I}+\mathbf{F}_{\mathbf{v}}{ }^{T} \otimes \mathbf{Y}\right) t}\left(x^{T} \otimes \mathbf{I}\right),
$$

where vec $\langle$ is the column stacking operator, which generates a column vector from the columns of a matrix.

Proof. The proof of the lemma is omitted due to space limitations.

$M_{\ell}^{(s)}$ is obtained by substituting the expressions (22) and (23) of the determined matrices $\mathbf{H}_{m, \ell}$ into the definition (2). For the generating function $M^{(s) *}(z)=$ $\sum_{\ell=0}^{\infty} z^{\ell} M_{\ell}^{(s)}$ we get

$$
\begin{aligned}
M^{(s) *}(z)=\sum_{\ell=0}^{\infty} z^{\ell} M_{\ell}^{(s)}= & -\underbrace{\sum_{\ell=0}^{\infty} z^{\ell} \int_{u=0}^{\infty} \sigma(u) \sum_{m=1}^{\infty} \beta \mathbf{P}_{m}^{(\mathbf{v})}(u) \Pi_{\mathbf{v s}} \mathbf{\Psi} \mathbf{S}^{m} \mathbf{R}^{\ell} \mathbb{1} d u}_{M_{1}^{(s) *}(z)} \\
& +\underbrace{\sum_{\ell=0}^{\infty} z^{\ell} \int_{u=0}^{\infty} \sigma(u) \sum_{m=1}^{\ell} \beta \mathbf{P}_{m}^{(\mathbf{v})}(u) \boldsymbol{\Pi}_{\mathbf{v s}} \mathbf{\Psi} \mathbf{R}^{\ell-m} \mathbb{1} d u}_{M_{3}^{(s) *}(z)} \\
& +\underbrace{\sum_{\ell=0}^{\infty} z^{\ell} \int_{u=0}^{\infty} \sigma(u) \sum_{m=\ell+1}^{\infty} \beta \mathbf{P}_{m}^{(\mathbf{v})}(u) \boldsymbol{\Pi}_{\mathbf{v s}} \boldsymbol{\Psi} \mathbf{S}^{m-\ell} \mathbb{1} d u}_{M_{2}^{(s) *}(z)} .
\end{aligned}
$$

By applying rearrangements and making use of Lemma 1 the above three terms can be expressed in closed-form resulting a formula for $M^{(s) *}(z)$ as

$$
\begin{aligned}
M^{(s) *}(z)= & \left(\beta \sigma^{*}\left(\mathbf{L}_{\mathbf{v}}+z \mathbf{F}_{\mathbf{v}}\right) \boldsymbol{\Pi}_{\mathbf{v s}} \mathbf{\Psi}-\operatorname{vec}^{T}\left\langle\boldsymbol{\Psi}^{T} \boldsymbol{\Pi}_{\mathbf{v s}}^{T}\right\rangle \sigma^{*}\left(\mathbf{L}_{\mathbf{v}}^{T} \otimes \mathbf{I}+\mathbf{F}_{\mathbf{v}}^{T} \otimes \mathbf{S}\right)\left(\beta^{T} \otimes \mathbf{I}\right)\right) \\
& \left((\mathbf{I}-z \mathbf{R})^{-1}+(z \mathbf{I}-\mathbf{S})^{-1} \mathbf{S}\right) \mathbb{1} .
\end{aligned}
$$


The generating function of the number of jobs in the system The phase $(\mathcal{J}(t))$ at the beginning of the cycles form an embedded discrete time Markov chain (DTMC). The probability matrices characterizing the number of arriving jobs and the phase transitions during the vacation period are $\int_{0}^{\infty} \sigma(x) \mathbf{P}_{m}^{(\mathbf{v})}(x) d x$. If $m$ jobs are in the queue when the system enters the service period then the phase transitions are given by $\mathbf{G}^{m}$, where matrix $\mathbf{G}$ is the minimal non-negative solution to the matrix-quadratic equation $\mathbf{0}=\mathbf{B}_{\mathbf{s}}+\mathbf{L}_{\mathbf{s}} \mathbf{G}+\mathbf{F}_{\mathbf{s}} \mathbf{G}^{2}$. Thus, the transition probability matrix of the DTMC, denoted by $\mathbf{Q}$, is expressed by

$$
\mathbf{Q}=\int_{0}^{\infty} \sigma(x) \sum_{m=0}^{\infty} \mathbf{P}_{m}^{(\mathbf{v})}(x) \boldsymbol{\Pi}_{\mathbf{v s}} \mathbf{G}^{m} \boldsymbol{\Pi}_{\mathbf{s v}} d x
$$

The stationary distribution of $\mathbf{Q}$, denoted by $\beta$, is determined by the linear system $\beta \mathbf{Q}=\beta, \beta \mathbb{1}=1$. Making use of Lemma 1 , vector $\beta$ is the solution to

$$
\operatorname{vec}^{T}\left\langle\boldsymbol{\Pi}_{\mathbf{v s}}^{T}\right\rangle \sigma^{*}\left(\mathbf{L}_{\mathbf{v}}^{T} \otimes \mathbf{I}+\mathbf{F}_{\mathbf{v}}^{T} \otimes \mathbf{G}\right)\left(\beta^{T} \otimes \boldsymbol{\Pi}_{\mathbf{s v}}\right)=\beta, \quad \beta \mathbb{1}=1 .
$$

Theorem 1. The generating function of the stationary number of jobs in the system, $q(z)$, is given by

$$
\begin{aligned}
q(z)=\frac{1}{c}( & \beta\left(\mathbf{I}-\sigma^{*}\left(\mathbf{L}_{\mathbf{v}}+z \mathbf{F}_{\mathbf{v}}\right)\right)\left(-\mathbf{L}_{\mathbf{v}}-z \mathbf{F}_{\mathbf{v}}\right)^{-1} \mathbb{1} \\
+ & \left(\beta \sigma^{*}\left(\mathbf{L}_{\mathbf{v}}+z \mathbf{F}_{\mathbf{v}}\right) \boldsymbol{\Pi}_{\mathbf{v s}} \mathbf{\Psi}-v e c^{T}\left\langle\mathbf{\Psi}^{T} \mathbf{\Pi}_{\mathbf{v s}}{ }^{T}\right\rangle \sigma^{*}\left(\mathbf{L}_{\mathbf{v}}^{T} \otimes \mathbf{I}+\mathbf{F}_{\mathbf{v}}^{T} \otimes \mathbf{S}\right)\left(\beta^{T} \otimes \mathbf{I}\right)\right) \\
& \left.\cdot\left((\mathbf{I}-z \mathbf{R})^{-1}+(z \mathbf{I}-\mathbf{S})^{-1} \mathbf{S}\right) \mathbb{1}\right),
\end{aligned}
$$

where $\beta$ is determined by (27) and the constant c satisfies $\lim _{z \rightarrow 1} q(z)=1$.

Taking the derivatives of $q(z)$ at $z \rightarrow 1$ provides the factorial moments of the number of jobs in the queue.

\section{Numerical example}

This numerical example investigates the effect of the mean and the distribution of the vacation time on the mean number of jobs in the system ${ }^{1}$. Since during the service period the arrival and the service processes are dependent we characterize the overall effect of the Markov environment by the following matrices :

$$
\begin{aligned}
& \mathbf{B}_{\mathbf{s}}=\left[\begin{array}{lll}
0 & 0 & 0 \\
1 & 0 & 1 \\
4 & 1 & 2
\end{array}\right], \mathbf{L}_{\mathbf{s}}=\left[\begin{array}{ccc}
-8 & 1 & 0 \\
0 & -5 & 2 \\
1 & 3 & -11
\end{array}\right], \mathbf{F}_{\mathbf{s}}=\left[\begin{array}{lll}
2 & 1 & 4 \\
0 & 1 & 0 \\
0 & 0 & 0
\end{array}\right], \\
& \mathbf{F}_{\mathbf{v}}=\left[\begin{array}{ll}
3 & 1 \\
0 & 1
\end{array}\right], \mathbf{L}_{\mathbf{v}}=\left[\begin{array}{cc}
-5 & 1 \\
2 & -3
\end{array}\right], \boldsymbol{\Pi}_{\mathbf{s v}}=\left[\begin{array}{cc}
1 & 0 \\
1 & 0 \\
0.1 & 0.9
\end{array}\right], \boldsymbol{\Pi}_{\mathbf{v s}}=\left[\begin{array}{ccc}
0.8 & 0 & 0.2 \\
0 & 0.7 & 0.3
\end{array}\right] .
\end{aligned}
$$

\footnotetext{
${ }^{1}$ The Mathematica implementation can be downloaded from http://hit.bme.hu/ qhorvath/software
} 


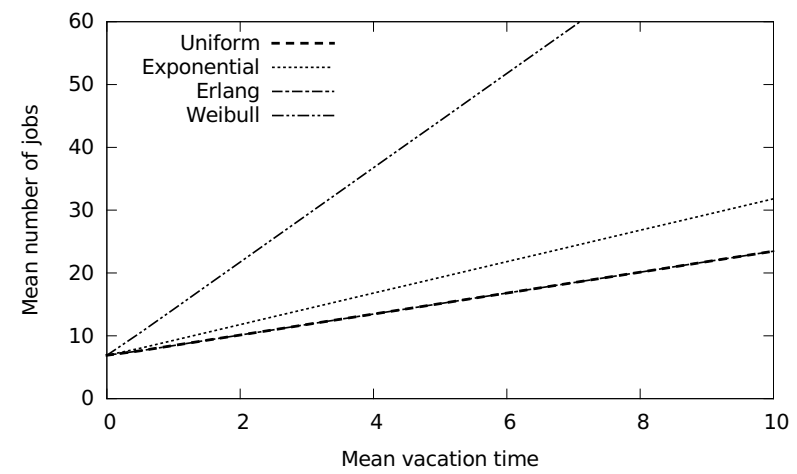

Fig. 2. The mean number of jobs in the system

The computation has been performed for the following type of vacation distributions: Uniform distribution, Exponential distribution, Erlang distribution with shape parameter of 3 , Weibull distribution with shape parameter of $k=1 / 2$. The mean number of jobs computed from Theorem 1 is depicted in Figure 2. As expected, the number of jobs in the system is the highest when the vacation times are Weibull distributed which has the heaviest tail. The plots corresponding to the uniform and the Erlang cases match completely. These distributions have the same squared coefficient of variations (that is $1 / 3$ ), thus the results suggest same kind of insensitivity as in the $\mathrm{M} / \mathrm{G} / 1$ queue.

\section{References}

1. Alfa, A.S.: A discrete $\mathrm{MAP} / \mathrm{PH} / 1$ queue with vacations and exhaustive timelimited service. Oper. Res. Lett. 18, 31-40 (1995)

2. Alfa, A.S.: Discrete time analysis of $\mathrm{MAP} / \mathrm{PH} / 1$ vacation queue with gated timelimited service. Queueing Systems 29(1), 35-54 (1998)

3. C. Goswami, N.S.: The discrete-time MAP/PH/1 queue with multiple working vacations. Applied Mathematical Modelling 34, 931-946 (2010)

4. Chang, S., Takine, T.: Factorization and stochastic decomposition properties in bulk queues with generalized vacations. Queueing Systems 50(2-3), 165-183 (2005)

5. Ke, J.C., Wu, C.H., Zhang, Z.G.: Recent developments in vacation queueing models: a short survey. International Journal of Operations Research 7(4), 3-8 (2010)

6. Latouche, G., Ramaswami, V.: Introduction to matrix analytic methods in stochastic modeling, vol. 5. Siam (1999)

7. Neuts, M.F.: A versatile markovian point process. Journal of Applied Probability pp. 764-779 (1979)

8. Saffer, Z., Telek, M.: Analysis of $B M A P / G / 1$ vacation model of non- $M / G / 1$-type. In: EPEW. LNCS, vol. 5261, pp. 212-226. Springer, Mallorca, Spain (2008)

9. Saffer, Z., Telek, M.: Closed form results for BMAP/G/1 vacation model with binomial type disciplines. Publ. Math. Debrecen 76(3), 359-378 (2010)

10. Tian, N., Zhang, Z.G.: Vacation queueing models: theory and applications, vol. 93. Springer Science \& Business Media (2006) 